\title{
A Multi-agent System to Learn from Oceanic Satellite Image Data
}

\author{
Rosa Cano, Angélica González, Juan F. de Paz, and Sara Rodríguez \\ Departamento Informática y Automática, Universidad de Salamanca \\ Plaza de la Merced s/n, 37008, Salamanca, Spain \\ \{rcano, angelica, fcofds, srg\}@usal.es
}

\begin{abstract}
This paper presents a multiagent architecture constructed for learning from the interaction between the atmosphere and the ocean. The ocean surface and the atmosphere exchange carbon dioxide, and this process is modeled by means of a multiagent system with learning capabilities. The proposed multiagent architecture incorporates CBR-agents to monitor the parameters that affect the interaction and to facilitate the creation of models. The system has been tested and this paper presents the results obtained.
\end{abstract}

Keywords: CBR-BDI, Air-Sea, Monitoring, Evaluation.

\section{Introduction}

Agents and multiagent systems are adequate for developing applications in dynamic, flexible environments. Agents can be characterized through their capacities in areas such as autonomy, communication, learning, goal orientation, mobility, persistence, etc. Autonomy, learning and reasoning are especially important aspects for an agent. These capabilities can be modelled in different ways and with different tools [19]. One of the possibilities is the use of Case Based Reasoning (CBR) systems. This paper presents a CBR based deliberative agent that incorporates neural networks to implement the retrieve, reuse, revise and retain stages of the CBR system. The CBR-BDI agent [7] is the core of a distributed system which mission is to monitor the interaction between the ocean surface and the atmosphere and learn from the data available. Initially the system has been used to evaluate and predict de quantity of $\mathrm{CO}_{2}$ exchanged in the North Atlantic Ocean by means of mining the data coming from satellite observations. The aim of this work is to obtain an architecture that makes it possible to construct dynamic systems capable of growing in dimension and adapting its knowledge to environmental changes. Several architectures have been proposed for building deliberative agents, most of them based on the BDI model. In the BDI model the internal structure of an agent and therefore its ability to choose a course of action is based on mental attitudes. The advantage of using mental attitudes in the design and realization of agents and multi-agent systems is the natural (human-like) modelling and the high abstraction level. The BDI (Beliefs, Desires, Intentions) model uses Beliefs as information attitudes, Desires as motivational attitudes and Intentions as deliberative attitudes 
for each agent. The method proposed in [4] facilitates the CBR systems incorporation as a reasoning engine in BDI agents, which makes it possible for an agent to have at its disposal a learning, adaptation and a greater degree of autonomy than a pure BDI architecture [4]. BDI agents can be implemented by using different tools. One very interesting tool is Jadex [16], a BDI reasoning engine that can be used on top of different middleware infrastructures such as JADE [5]. Jadex agents deal with the concepts of beliefs, goals and plans. Beliefs, goals and plans are objects that can be created and handled within the agent at execution time. Jadex has the advantage of allowing programmers to include their own deliberative mechanisms. In this case this mechanism will be a CBR system. Moreover the system will benefit from all the communication advantages that JADE provides.

In the next section we review the environmental problem that motivates most of this research. Section three describes the CBR-BDI agent based system developed. Finally the conclusions and some preliminary results are presented.

\section{Air Sea Interaction Problem}

In recent years a great interest has emerged in climactic behaviour and the impact that mankind has had on the climate. One of the most worrying factors is the quantity of $\mathrm{CO}_{2}$ present in the atmosphere. Until only a few years ago, the photosynthesis and breathing processes in plants were considered as the regulatory system that controls the presence of $\mathrm{CO}_{2}$ in the atmosphere. However, the role played by the ocean in the regulation of carbon volume is very significant and so far remains indefinite [17]. Current technology offers the possibility of obtaining data and estimates that were beyond expectations only a few years ago. The goal of this project is to construct a model that calculates the global air-sea flux of $\mathrm{CO}_{2}$ exchanged between the atmosphere and the surface waters of the ocean. In order to create a new model for the $\mathrm{CO}_{2}$ exchange between the atmosphere and the oceanic surface a number of important parameters must be taken into consideration: sea surface temperature, air temperature, sea surface salinity, atmospheric and hydrostatic pressures, the presence of nutrients and the wind speed vector (module and direction) [18].

These parameters can be obtained from oceanographic ships as well as from satellite images. Satellites provide a great amount of daily information and there is a growing need of automatically process and learn from this source of knowledge. These parameters allow us to calculate the variables that define our models, such as the velocity of gas transfer, solubility, or the differentiation between partial pressures on the atmosphere and sea surface (a case structure is shown in Table 1).

Satellite information is vital for the construction of oceanographic models, and in this case, in order to produce estimates of air-sea fluxes of $\mathrm{CO}_{2}$ with much higher spatial and temporal resolution, using artificial intelligence models than can be achieved realistically by direct in situ sampling of upper ocean $\mathrm{CO}_{2}$. In order to handle all the potentially useful data to create daily models in reasonable time and at a reasonable cost, it is necessary to use automated distributed systems capable of incorporating new knowledge. Our proposal is presented in the following section. 


\section{CBR-BDI Modelling Agent}

Our final aim is to model both, deal with data obtained from the open ocean and shelf seas, and it is believed that by assimilating Earth Observation (EO) data into artificial intelligence models these problems may be solved. Earth observation data (both for assimilation and for validation) are vital for the successful development of reliable models that can describe the complex physical and biogeochemical interactions involved in marine carbon cycling. Satellite information is vital for the construction of oceanographic models, and in this case, to produce estimates of air-sea fluxes of carbon dioxide with much higher spatial and temporal resolution, using artificial intelligence models than can be achieved realistically by direct in situ sampling of upper ocean carbon dioxide. To handle all the potentially useful data to create daily models in a reasonable time and with a reasonable cost, it is necessary to use automated distributed systems capable of incorporating new knowledge. Our proposal consists of a multiagent system which main characteristic is the use of CBR-BDI agents.

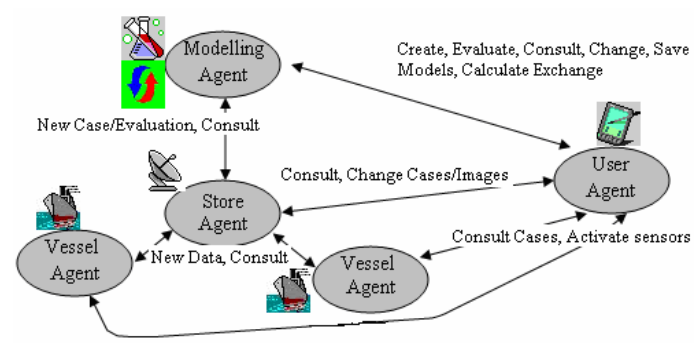

Fig. 1. Diagram of the architecture of our MAS

Figure 1 illustrates a multiagent system in which is it possible to observe how a Modelling agent with a CBR-BDI architecture is responsible for the creation and evaluation of models in terms of the data received from the Store, Vessel and User agents. This model allows us to monitor and predict the carbon dioxide exchange between the ocean surface and the atmosphere. The Store agent processes the images from the satellite and transforms them for use by the system. Each Vessel agent is installed in a ship and collects information in-situ that allows us to evaluate the models created by the Modelling agent. The User agent can interact with any of the other agents. Figure 1 shows how the agents interact with each other and with their surroundings. From the oceanographic point of view, in order to resolve the problem that confronts us, the ocean has been divided into a series of zones. In each of these zones there will be a Modelling Agent, a Store Agent, and various Vessel Agents.

Figure 2 presents the class diagram for the Modelling agent that has two principal functions. The first is to generate models which are capable of predicting the atmospheric/oceanic interaction in a particular area of the ocean in advance. The second is 


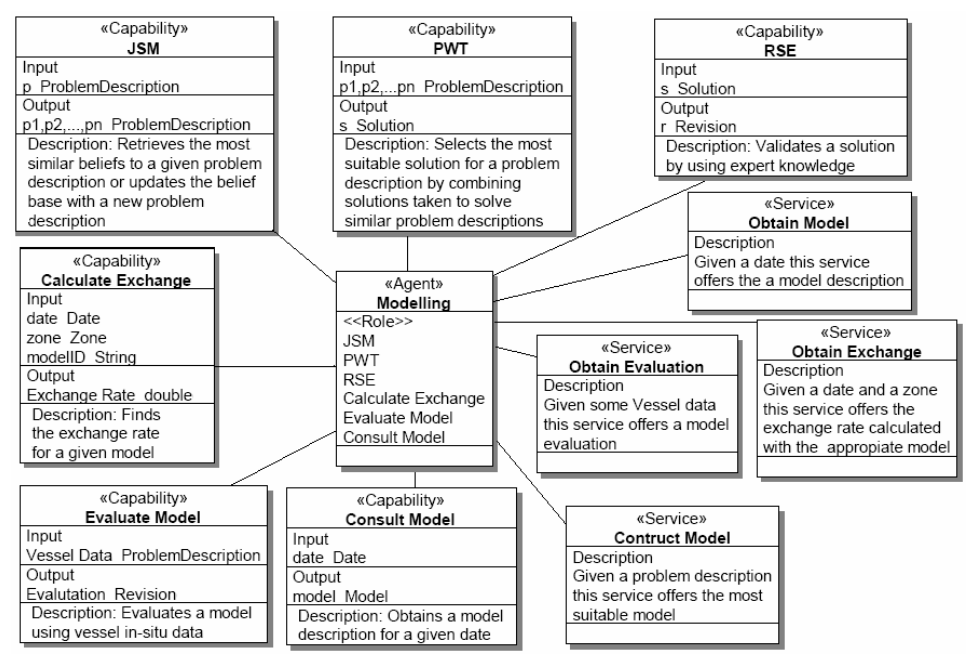

Fig. 2. Class diagram for the Modelling agent

to permit the use of such models. The reasoning cycle of a CBR system is included among the activities, composed of stages of retrieval, reuse, revise and retain. Also, an additional stage is used that introduces expert's knowledge. This reasoning cycle must correspond to the sequential execution of some of the agent roles. The Modelling agent carries out roles to generate models such as Jacobean Sensitivity Matrix (JSM), Pondered Weigh Technique (PWT), Revision Simulated Equation (RSE), and other roles that allow it to operate with the models calculated, like Forecast Exchange Rate, Evaluate Model or Consult model. The roles used to carry out the stages of the CBR cycle are now described.

Jacobean Sensitivity Matrix (JSM): This role is in charge of carrying out the retrieval stage. In order to do this it needs to use a method that guarantees the recuperation of cases whose characteristics are similar to the current problem. The Jacobean Sensitivity Matrix (JSM) is used in this case for data clustering and retrieval [15]. The Jacobean Sensitivity Matrix method is a novel approach for feature selection. It can be used to visualize and extract information from complex, and highly dynamic data. The model is based in the principal component analysis and is used to identify which input variables have more influence in the output of the neural network used to perform the principal component analysis. The neural network identifies the beliefs stored by the agent that can be more useful to solve a given problem. The mathematical model is now outlined.

If JSM is a matrix NxM where $N$ is the number of input of the neural network and $M$ is the number of output of the neural network. And if the element $S_{k i}$ in the matrix represents the sensitivity (influence) of the output $k$ over the input $I$, then (1).

$S_{k i}=\frac{\partial y_{k}}{\partial x_{i}}=\frac{\partial f_{k}\left(n e t_{k}\right)}{\partial x_{i}}=\frac{\partial f_{k}\left(n e t_{k}\right)}{\partial n e t_{k}} \frac{\partial n e t_{k}}{\partial y_{j}} \frac{\partial y_{j}}{\partial n e t_{j}} \frac{\partial n e t_{j}}{\partial x_{i}}=\frac{\partial f_{k}\left(n e t_{k}\right)}{\partial n e t_{k}}\left(\sum_{j=1}^{H} w_{k j} \frac{\partial f_{j}\left(n e t_{j}\right)}{\partial n e t_{j}} w_{j i}\right)$ 
Where $w_{i j}$ is the weight of the connection between the input neuron $i$ and the hidden neuron $j$. $w_{k j}$ is the weight of the connection between the hidden neuron $j$ and the output neuron $k . y_{k}$ is the output obtained for neuron $k$ of the output layer. Then $y_{k}=f_{k}$ $\left(n e t_{k}\right) \cdot y_{j}$ is the output obtained for neuron $j$ of the hidden layer. Then $y_{j}=f_{j}\left(n e t_{j}\right) . x_{i}$ is the input for neuron $i$ and $f_{h}$ is the activation function in neuron $h$. Then

$$
\begin{aligned}
& n e t_{j}=\sum_{i=1}^{N} w_{j i} x_{i}+\theta_{j} \\
& n e t_{k}=\sum_{j=1}^{H} w_{k j} y_{j}+\theta_{k}
\end{aligned}
$$

Where $H$ is the number of neurons in the hidden layer, $\theta_{j}$ is the value of threshold of neuron $j$ of the hidden layer and $\theta_{k}$ is the value of threshold of neuron $k$ of the output layer.

Pondered Weigh Technique (PWT): The reuse is carried out using the cases selected during the retrieval stage. The cases are pondered [13] and the bigger weight is given to the one that more resembles the current problem in the following way:

$$
p^{*}=\frac{1}{\sum_{r=1}^{Z} e^{-|a-r|}} \sum_{r=1}^{Z} e^{-|a-r|} p^{r}
$$

Where $p^{*}$ is the solution prediction, $Z$ is the number of retained cases from the base of beliefs, $a$ is the measure of minimum similarity between the retained cases from the base of beliefs and the current case, $p^{r}$ is the retained prediction $\mathrm{r}$-th from the base of beliefs and $r$ is the measure of similarity between the retained cases $r$-th from the base of beliefs and the current case.

Revision Simulated Equation (RSE): During the revision stage an equation $(\mathrm{F})$ is used to validate the proposed solution $p^{*}$.

$$
F=k s o\left(p C O_{2} S W-p C O_{2} A I R\right)
$$

Where $\mathrm{F}$ is the flux of $\mathrm{CO}_{2}, k$ is the gas transfer velocity (6), so is the solubility verifying (7) and $\mathrm{pCO}_{2}$ is the partial pressure of $\mathrm{CO}_{2}(8)$.

$$
\begin{gathered}
k=(-5,204 \text { Lat }+0,729 \text { Long }+2562,765) / 3600 \\
\text { so }=e^{\left(\frac{93,4517}{100 t k}-60,2409+23,3585 \log (100 t k)+s(0,023517-0,023656 \bullet 100 t k+0,0047036 \bullet 1002 t k)\right)} \\
p C \mathrm{O}_{2}=A+\text { BLong }+ \text { CLat }+ \text { DSST }+ \text { EYear }
\end{gathered}
$$

As can be seen in (6), $k$ depends on Lat (Latitude), Long (Longitude). As can be seen in (7) $s o$ depends on $t k=273,15+t$. where $t$ is the temperature and $s$ is the salinity. Finally, in (8) it is possible to observe that $\mathrm{pCO}_{2}$ depends on the SST. SST is the temperature of the marine surface or air as it corresponds to $\mathrm{pCO}_{2} \mathrm{SW}$ or $\mathrm{pCO}_{2} \mathrm{AIR}$. The coefficients of the equation (8) depend on the month. 
Table 1. Case Attributes

\begin{tabular}{ll}
\hline Case Field & Measurement \\
\hline DATE & Date $(\mathrm{dd} / \mathrm{mm} /$ yyyy) \\
LAT & Latitude (decimal degrees) \\
LONG & Longitude $($ decimal degrees) \\
SST & Temperature $\left({ }^{\circ} \mathrm{C}\right)$ \\
S & Salinity (unitless) \\
WS & Wind strength $(\mathrm{m} / \mathrm{s})$ \\
WD & Wind direction (unitless) \\
Fluo_calibrated & fluorescence calibrated with chlorophyll \\
SW pCO $\mathrm{CO}_{2}$ & surface partial pressure of $\mathrm{CO}_{2}$ (micro Atmospheres) \\
Air pCO $\mathrm{CO}_{2}$ & air partial pressure of $\mathrm{CO}_{2}($ micro Atmospheres) \\
Flux of $\mathrm{CO}_{2}$ & $\mathrm{CO}_{2}$ exchange flux $\left(\mathrm{Moles} / \mathrm{m}^{2}\right)$ \\
\hline
\end{tabular}

During the revision, the agent compares the obtained $\mathrm{F}$ value with predicted one and if the prediction differs in less than $10 \%$ the case is stored on the base of beliefs. As has been shown the CBR-BDI agents use a CBR system, at a low level of implementation, which is the reason for using cases. One case for the CBR consists of a problem (initial situation and a number of goals) and the plans to resolve it. For oceanic/atmospheric interaction, we define the problem in terms of the attributes shown in Table 1.

Table 1 shows the description of a case: DATE, LAT, LONG, SST, S, WS, WD, Fluo_calibrated, $\mathrm{SW} \mathrm{pCO}$ and Air $\mathrm{pCO}_{2}$. Flux of $\mathrm{CO}_{2}$ is the value to be identified.

\section{Results and Conclusions}

The application of Artificial Intelligence techniques is extremely useful in a field like oceanography and specifically in the study of the carbon dioxide exchange between the ocean surface and the atmosphere. The use of CBR-BDI agents facilitates dealing with a great amount of satellite images.

The system described above was tested in the North Atlantic Ocean during 2005. Although the system is not fully operational and the aim of the project is to construct a research prototype and not a commercial tool, the initial results have been very successful from the technical and scientific point of view. The construction of the distributed system has been relatively simple using previously developed CBR-BDI libraries $[2,3,6,7,8,10]$. The formalism defined in [7] facilitates the straight mapping between the agent definition and the CBR construction. The multiagent system has automatically incorporated over 50,000 instances during the five months and eliminated $12 \%$ of the initial ones.

Figure 3 shows the appearance of a Vessel agent. The vessel agent periodically sends information about the data obtained from its corresponding ship. The in-situ data obtained as well as the route followed by the ship can be easily observed by the oceanographers by means of their user agent. For this study we have used vessel simulators, which work with real stored data. 

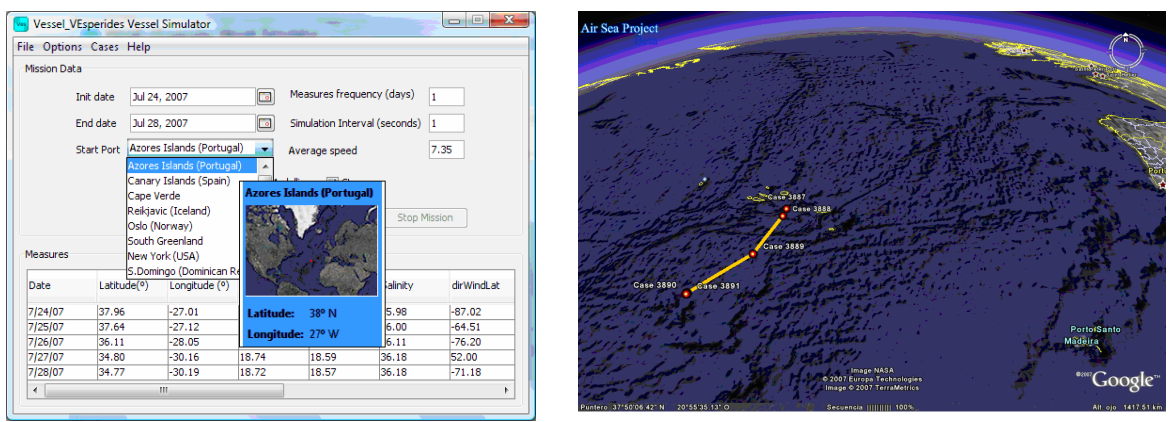

Fig. 3. Vessel agent interface (a) and presentation of the routed followed for a ship by means of the Google Eath tool (b)

Table 2. Million of tones of $\mathrm{CO}_{2}$ exchanged in the North Atlanthic

\begin{tabular}{llllll}
\hline & Oct. 05 & Nov. 05 & Dec. 06 & Jan. 06 & Feb. 06 \\
\hline Multiagent System & -19 & 21 & 33 & 29 & 29 \\
Manual models & -20 & 25 & 40 & 37 & 32 \\
\hline
\end{tabular}

The system has been tested during the last three months of 2005 and the results have been very accurate. Table 2 presents the results obtained with the Multiagent systems and with mathematical Models [14] used by oceanographers to identify the amount of $\mathrm{CO}_{2}$ exchanged. The numerical values represent the million of Tonnes of carbon dioxide that have been absorbed (negative values) or generated (positive value) by the ocean during each of the three months.

The values proposed by the CBR-BDI agent are relatively similar to the ones obtained by the standard technique. While the CBR-BDI Modelling Agent generates results on a daily basis without any human intervention, the Casix manual modelling techniques require the work of one researcher processing data during at least four working days. Although the system proposed requires further improvements and more work the initial results are very promising. Compared to the previously CBR-BDI models developed based on Hebbian Learning (CoHel) $[1,6,10]$ or variational calculus techniques (VCBP) [2,9], the results obtained with the reasoning engine presented in this paper are very similar to those obtained applying hebbian learning and give a quicker response than VCBP engines. This work present the development of new algorithms to improve the CBR engine incorporated in the BDI agent. These algorithms are included in each of the stages of the CBR reasoning cycle.

\section{References}

1. Bajo, J., Corchado, J.M.: Evaluation and monitoring of the air-sea interaction using a CBR-Agents approach. In: Muñoz-Ávila, H., Ricci, F. (eds.) ICCBR 2005. LNCS (LNAI), vol. 3620, pp. 50-62. Springer, Heidelberg (2005)

2. Bajo, J., Corchado, J.M., de Paz, Y., de Paz, J.F., Rodríguez, S., Martín, A., Abraham, A.: SHOMAS: Intelligent Guidance and Suggestions in Shopping Centres. Applied Soft Computing 9(2), 851-862 (2009) 
3. Bajo, J., de Paz, J.F., de Paz, Y., Corchado, J.M.: Integrating Case-based Planning and RPTW Neural Networks to Construct an Intelligent Environment for Health Care. Expert Systems with Applications, Part 2 36(3), 5844-5858 (2009)

4. Bratman, M.E.: Intentions, Plans and Practical Reason. Harvard University Press, Cambridge (1987)

5. Bellifime, F., Poggi, A., Rimasa, G.: JADE: a FIPA2000 compliant agent development environement. In: Proceedings of the 5th international conference on autonomous agents. ACM, New York (2001)

6. Corchado, J.M., Lees, B.: A Hybrid Case-based Model for Forecasting. Applied Artificial Intelligence 15(2), 105-127 (2001)

7. Corchado, J.M., Laza, R.: Constructing Deliberative Agents with Case-based Reasoning Technology. International Journal of Intelligent Systems 18(12), 1227-1241 (2003)

8. Corchado, J.M., Bajo, J., Abraham, A.: GERAmI: Improving the delivery of health care. IEEE Intelligent Systems 23(2), 19-25 (2008)

9. Corchado, J.M., Bajo, J., de Paz, Y., Tapia, D.: Intelligent Environment for Monitoring Alzheimer Patients, Agent Technology for Health Care. Decision Support Systems 34(2), 382-396 (2008)

10. Corchado, J.M., Aiken, J., Corchado, E., Lefevre, N., Smyth, T.: Quantifying the Ocean's CO2 Budget with a CoHeL-IBR System. In: Funk, P., González Calero, P.A. (eds.) ECCBR 2004. LNCS (LNAI), vol. 3155, pp. 533-546. Springer, Heidelberg (2004)

11. Dransfeld, S., Tatnall, A.R., Robinson, I.S., Mobley, C.D.: A comparison of Multi-layer Perceptron and multilinear regression algorithms for the inversion of synthetic ocean colour spectra. Int. J. Remote Sens. 25(21), 4829-4834 (2004)

12. Dransfeld, S., Tatnall, A.R., Robinson, I.S., Mobley, C.D.: Prioritizing ocean colour channels by neural network input reflectance perturbation. Int. J. Remote Sens. 26(5), 1043$1048(2005)$

13. De Paz, Y.D.R.: Mixture Weibull distribution using artificial neural networks with censurated data PHD thesis, ch. 3 (2005)

14. Lefevre, N., Aiken, J., Rutllant, J., Daneri, G., Lavender, S., Smyth, T.: Observations of pCO2 in the coastal upwelling off Chile: Sapatial and temporal extrapolation using satellite data. Journal of Geophysical research 107 (2002)

15. Montaño, J.J., Palmer, A.: Artificial Neural Networks, opening the black box. Metodología de las Ciencias del Comportamiento 4(1), 77-93 (2002)

16. Pokahr, A., Braubach, L., Lamersdorf, W.: Jadex: Implementing a BDI-Infrastructure for JADE Agents. EXP - In Search of Innovation (Special Issue on JADE) 3(3), 76-85 (2003)

17. Santamaría, J., Nieto, J.: Los agujeros del cambio climático. World Watch (12), 62-65 (2000)

18. Takahashi, T., Olafsson, J., Goddard, J.G., Chipman, D.W., Sutherland, S.C.: Seasonal Variation of $\mathrm{CO} 2$ and nutrients in the High-latitude surface oceans: a comparative study. Global biochemical Cycles 7(4), 843-878 (1993)

19. Wooldridge, M., Jennings, N.R.: Agent Theories, Architectures, and Languages: a Survey. In: Wooldridge, M., Jennings, N.R. (eds.) Intelligent Agents, pp. 1-22. Springer, Heidelberg (1995) 